\title{
INTRODUCTION : LES RÉFORMES ET LES REGROUPEMENTS ADMINISTRATIFS EN EUROPE - QUESTIONS DE RECHERCHE ET DÉFIS EMPIRIQUES
}

\author{
Alistair Cole, Jean-Michel Eymeri-Douzans
}

I.I.S.A. | « Revue Internationale des Sciences Administratives »

2010/3 Vol. 76 | pages 423 à 434

ISSN 0303-965X

Article disponible en ligne à l'adresse :

https://www.cairn.info/revue-internationale-des-sciences-

administratives-2010-3-page-423.htm

Distribution électronique Cairn.info pour I.I.S.A..

(C) I.I.S.A.. Tous droits réservés pour tous pays.

La reproduction ou représentation de cet article, notamment par photocopie, n'est autorisée que dans les limites des conditions générales d'utilisation du site ou, le cas échéant, des conditions générales de la licence souscrite par votre établissement. Toute autre reproduction ou représentation, en tout ou partie, sous quelque forme et de quelque manière que ce soit, est interdite sauf accord préalable et écrit de l'éditeur, en dehors des cas prévus par la législation en vigueur en France. Il est précisé que son stockage dans une base de données est également interdit. 


\title{
Revue \\ Internationale \\ des Sciences \\ Administratives
}

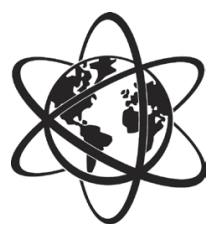

\section{Introduction : Les réformes et les regroupements administratifs en Europe \\ - Questions de recherche et défis empiriques}

\author{
Alistair Cole et Jean-Michel Eymeri-Douzans
}

Ce numéro spécial a vu le jour à la suite de deux ateliers organisés par le Groupe de travail «Science politique comparée des administrations » (SPCA) de l'Association française de science politique (AFSP) en avril et septembre 2008. Nos ateliers portaient sur une dimension relativement peu étudiée de l'administration publique comparée, à savoir les regroupements ou fusions d'administrations et les réorganisations bureaucratiques en tant que dimension des réformes contemporaines. Nous sommes partis du constat suivant : depuis quelques années, les ouvrages abondent sur le rôle des agences et les autres formes de décentralisation organisationnelle, mais ceux qui s'intéressent aux regroupements et fusions au sein des administrations publiques sont nettement plus rares. Les participants étaient invités à aborder un ensemble de questions connexes visant à essayer d'élucider la nature et l'ampleur des réorganisations administratives dans les pays d'Europe observés. Au nombre de ces questions, l'on trouvait : dans quelles conditions et dans quelle mesure les regroupements et autres formes de réorganisations administratives ont-ils des conséquences durables sur l'appareil d'État? De quelle manière la structure multi-niveaux de l'État, en particulier dans les États fédéraux (Allemagne, Belgique) et les États régionalisés (Espagne) ou « doubles» (Royaume-Uni), influence-t-elle les réformes administratives ? Sur quels récits de politique publique se fonde-t-on pour justifier et légitimer ces réorganisations, et plus particulièrement, quelle est l'influence du discours du nouveau management public (NMP) à cet égard ? Enfin, quelles sont les conséquences de ces réformes pour les carrières des agents de l'État, leurs identités professionnelles et les cultures administratives ? Ce numéro rassemble une série d'articles portant sur différents pays, qui ont pour ambition d'aborder ces questions et de contribuer à enrichir la réflexion comparative et empirique sur les dimensions et les formes de réformes de l'État à l'ère du NMP.

\footnotetext{
Alistair Cole est professeur à la School of European Studies, Université de Cardiff, Pays de Galles, Royaume-Uni, et Jean-Michel Eymeri-Douzans est professeur de Science politique à I'Institut d'études politiques de Toulouse, France. 


\section{Cadrage des recherches}

On établit souvent une distinction fondamentale entre les processus de spécialisation, dans lesquels les appareils bureaucratiques sont davantage différenciés et fragmentés, et les processus de « déspécialisation », qui sous-entendent une intégration structurelle d'organisations autrefois séparées. Contrairement au premier processus, les fusions et réorganisations administratives ont suscité assez peu d'intérêt chez les chercheurs. Le fait de jouer avec la «structure de l'État » et de redéfinir les bureaucraties est une activité aussi ancienne que la bureaucratie proprement dite, et les exemples célèbres sont nombreux à travers l'Histoire, tels ceux de Frédéric le Grand en Prusse, Napoléon ou Pierre le Grand de Russie. C'est peut-être l'une des raisons qui explique qu'on les considère comme un « vieil objet » dans le cadre d'un « vieil institutionnalisme », alors que les chercheurs se revendiquant du « néo-institutionnalisme », influencés par le « tournant cognitif » des sciences sociales, ont défini les institutions en termes de discours et de croyances (Schmidt, 2008), de dépendance au sentier (Peters, Pierre et King, 2005) ou de formes de « comportement approprié » (March et Olsen, 1989), et accessoirement seulement en tant qu'organisations. En conséquence, certains spécialistes en administration publique préfèrent, depuis deux décennies, s'intéresser à la nouveauté des idées et des prescriptions du nouveau management public (NMP) plutôt que de s'investir dans des analyses institutionnelles finement ancrées au plan empirique. D'où parfois une tendance à négliger les réformes administratives concrètes, comme les regroupements et fusions, les considérant comme un sous-produit de la diffusion mondiale du NMP plutôt que comme des objets de recherche à part entière. Contrairement à ces approches « par le haut » (top-down), les articles proposés dans ce numéro suivent une perspective davantage " par le bas » (bottom-up) et s'efforcent de prendre les réorganisations administratives au sérieux, et d'explorer de façon critique le degré auquel elles ont partie liée au NMP.

Le développement et la diffusion transnationale du NMP, d'abord dans les pays de l'OCDE puis partout dans le monde, ont été remarquables depuis le début des années 1980. Les ouvrages académiques sont nombreux qui démontrent que tous les gouvernements nationaux des pays développés ou presque, indépendamment de leur orientation à droite ou à gauche, ont adopté d'ambitieuses politiques de « réforme administrative » ou de «modernisation administrative ». Les réformes plus ou moins axées sur le NMP visaient explicitement non seulement à réduire les coûts des services publics (budgets publics, dette publique et impôts), mais aussi à améliorer la qualité des services publics, le résultat attendu étant un État de meilleure qualité : " un meilleur rapport qualité-prix » (" best value for (less) money ») ! Sur un certain plan, l'on peut voir le NMP comme une façon d'appliquer la logique et les méthodes du secteur privé pour améliorer les célèbres « trois E » (économie, efficience et efficacité) des services publics ; son association aux expériences de gouvernements néolibéraux, notamment au RU et en Nouvelle-Zélande, mais aussi aux États-Unis et au Canada, lui a rapidement conféré un tour idéologique marqué (Savoie, 1994). Les chercheurs en administration publique comparée ont néanmoins démontré que le NMP n'était pas un dogme fixe et systématique, mais un « paradigme » produit par syncrétisme (Hood, 1991 \& 1995), un mélange d'idées, de croyances, de valeurs, de slogans et de récits de politique publique venant en appui 
d'un répertoire pratique de recettes, de techniques et d'instruments managériaux : on peut même soutenir que le NMP est davantage une " praxéologique » qu'une idéologie (Eymeri-Douzans, 2010). Le contenu de ce répertoire néo-managérialiste comprend des éléments bien connus : agencification, benchmarking, indicateurs de performance, procédures d'accréditation, chartes citoyennes, transparence accrue, gestion par objectifs, rémunération à la performance, budgets de programmes, procédures d'imputabilité, fusions d'administrations et guichets uniques, administration électronique, partenariats public-privé et délégation de services publics à des entités privées, "gestion par la qualité totale », certification et évaluation ex ante, in itinere et ex post. Ces recettes et techniques sont souvent vantées telles des « meilleures pratiques » dans les forums internationaux et européens, comme en témoignent, par exemple, les différents programmes et réseaux axés sur la « bonne gouvernance » et les réformes administratives poussées par la Banque mondiale, le FMI, l'OCDE et I'UE. Les gouvernants de maints pays très différents ont sélectionné tout ou partie de cette « boîte à outils » du NMP et les ont transplantés/transposés dans leur propre administration publique (à propos des transferts de politiques en général, voir Dolowitz, 2000; De Jong et al., 2002). Cette dimension « praxéologique » du NMP est incontestablement sa plus grande force en termes de facilité de transfert : ceux qui sont au pouvoir dans nos différents États-nations, tant les gouvernants élus que les hauts fonctionnaires, ont importé et mis en œuvre la boîte à outils néo-managérialiste sans avoir à déclarer explicitement leur adhésion à l'ensemble idéologique néolibéral fondateur du NMP.

Mais ce caractère praxéologique du NMP est aussi une de ses principales faiblesses, car ce " paradigme » de politique publique (au sens de Hall, 1993) est utilisé d'un point de vue tactique pour justifier des réformes organisationnelles différentes, comme l'agencification et/ou les fusions administratives. Dans la pratique, cependant, spécialisation et déspécialisation peuvent être interprétées comme les deux faces d'un même phénomène ; il s'agit dans les deux cas d'essayer de donner un sens stratégique à des réformes institutionnelles et de résoudre les problèmes grandissants de coordination dans l'État contemporain. Les administrations se restructurent de différentes manières, qui vont de la réorganisation des ministères et de leurs directions générales à la création d'agences qui fragmentent la bureaucratie. À l'instar des agences, les regroupements administratifs s'accompagnent de plus en plus d'un discours managérial qui, dans ses différentes expressions et incantations, promeut cette approche comme relevant d'un sens commun adapté. L'« efficience » est sans aucun doute le maître-mot pour justifier les fusions, aussi bien que pour rationaliser les scissions et les spécialisations organisationnelles, et plus généralement pour justifier toute orientation de politique publique à notre ère managérialiste. En s'attaquant à l'enjeu de la coordination et en limitant les chevauchements entre échelons bureaucratiques réputés redondants, les regroupements administratifs sont censés rendre l'État plus efficace. Compte tenu de l'accent mis sur la réduction des dépenses publiques, les regroupements (encore une fois, tout comme l'agencification) sont souvent considérés comme une recette efficace pour réduire la bureaucratie, tout en permettant d'importantes économies d'échelle en matière de personnel, de locaux ou de logistique. Les articles rassemblés dans ce numéro spécial invitent par conséquent le lecteur à examiner sous l'angle critique ce discours néo-managérialiste employé « en toutes saisons » pour justifier des mesures contradictoires 
au moyen du même ensemble d'arguments. Conformément aux conclusions de la grande enquête menée par Bouckaert et Pollitt (2004), les articles présentés dans ce numéro spécial jettent un doute sur la convergence des formes organisationnelles dans un sens qui confirmerait la thèse d'une « néo-managérialisation » homogène en dépit des particularités nationales, locales et sectorielles. II convient de souligner que, même dans les limites d'un seul pays, les styles organisationnels varient au fil du temps et d'un secteur d'action publique à l'autre.

Ce numéro spécial présente ainsi une sélection d'études de cas européens, analysés dans une perspective européenne comparative afin de mieux comprendre ce qui est en jeu concrètement dans ces réorganisations administratives. Le choix des cas offre une variété certes limitée mais pourtant suffisante, ce qui permet de tirer quelques conclusions comparatives, limitées aux États démocratiques d'Europe occidentale. Tous les États examinés ici sont membres de l'Union européenne, et sont donc tous potentiellement concernés par des interactions multi-niveaux. Les États étudiés comprennent des États unitaires, fédéraux et régionalisés ; ils comprennent aussi des États aux traditions administratives napoléoniennes, aux traditions allemandes de Rechtstaat, ainsi que certains États aux régimes plus libéraux. Leurs élites administratives comprennent des administrateurs généralistes, des experts spécialisés, des membres de " grands corps » techniques prestigieux et des représentants de la profession juridique. En somme, nos exemples couvrent une série de comparateurs logiques en Europe occidentale. Si l'accent est mis sur « le cœur de l'Exécutif » (au sens défini par Rhodes, 1995), dans le cas de l'Espagne, la définition de ce cœur est élargie pour prendre en considération le développement de formes asymétriques d'administration publique territoriale : dans ce cas particulier, les questions de réorganisations administratives sont reliées au problème plus large de la reconfiguration territoriale de l'État.

\section{Des similarités fondamentales... et des différences essentielles}

Même si chaque article examine un cas national défini, ces articles invitent dans leur ensemble à une réflexion plus large sur l'influence du NMP et les modalités de réformes de l'État en Europe au vu de la réalité des regroupements administratifs. Sur ce fondement, il semble possible d'identifier (au moins) cinq variables agissantes essentielles, qui influencent les pressions exogènes (internationales) et endogènes (localisées) en faveur du changement et les cas empiriques de restructuration administrative. Ces variables sont : les héritages institutionnels, les effet de mode en matière de réforme, les logiques hybrides d'ingénierie institutionnelle, l'interaction entre les stratégies collectives des institutions et groupes concernés, et enfin les dynamiques multi-niveaux.

L'importance des « héritages » du passé (Pierson, 2004) est un sujet bien documenté dans la littérature, qu'il soit conceptualisé en termes de trajectoires nationales « d'étaticité » (Linz \& Stepan, 1996), d'influence de la structure du gouvernement, ou de caractère duratif des « styles d'action publique » (Richardson \& Jordan, 1982) et des héritages ou legs de programmes publics (voir notamment les études classiques de Rose et Davies, 1994). Dans les cas examinés ici, les héritages institutionnels sont le facteur le plus apparent de résilience des structures administratives nationales et des configurations interinstitutionnelles face aux pressions néomanagériales 
transnationales en faveur du changement. Parmi les diverses études de cas présentées, la persistance d'arrangements institutionnels nationaux traditionnels est frappante en Allemagne (où le principe d'autonomie ministérielle reste primordial), dans une certaine mesure en France (où les corps, ces organes administratifs, manoeuvrent pour sauver leur existence et conserver leur influence), et même au Royaume-Uni (où l'agencification, contrairement à ce que l'on affirme souvent, n'a pas été le principal problème pour le rôle des hauts fonctionnaires). En Espagne, l'important défi à l'autorité centrale lancé par d'ambitieux gouvernements infra-nationaux assoiffés d'autonomie l'a emporté sur les réorganisations administratives du gouvernement central.

Rien d'étonnant à ce que la tradition de départementalisation reste une caractéristique importante de l'architecture du secteur public partout en Europe. Du point de vue de l'institutionnalisme historique, le processus considéré par Weber comme une différenciation/spécialisation des tâches de l'État a laissé une empreinte génétique marquée. Dans tous les États membres, on trouve une tendance héritée commune, caractérisée par un exécutif central divisé en départements et ministères, eux-mêmes subdivisés en directions générales (directions d'administration centrale, divisions, Abteilung). Les réformes administratives mises en œuvre ces dernières décennies, même si elles sont parfois conçues pour des instruments ou des agencements alternatifs à ce qui l'on perçoit comme les hiérarchies et les limites trop rigides de l'État wébérien sectorisé, n’ont nulle part entraîné la disparition des ministères sectoriels, qui restent une caractéristique solide de tout appareil d'État dans l'Europe d'aujourd'hui.

Le cas du Royaume-Uni illustre bien jusqu'où les tentatives de réforme peuvent... et ne peuvent pas aller. Le principe organisationnel fondamental au sein de l'Etat britannique (depuis le comité Haldane de 1918) est celui des départements ministériels et de la doctrine de la responsabilité ministérielle individuelle qui, en théorie, crée des lignes de responsabilité bien définies. À différents stades dans l'histoire britannique récente, cependant, ce modèle a été attaqué, les réformateurs soutenant que la départementalisation créait des silos, entraînait un gaspillage de ressources et encourageait une mentalité de "bureau-shaping», qui conduit les agences à se décharger des problèmes sur d'autres agences publiques ou sur les gouvernements locaux. Les initiatives visant à réformer l'exécutif central étaient centrées sur le Premier ministre. Par exemple, le Premier ministre conservateur Heath a tenté de regrouper une série de petits départements en des ministères plus vastes en 1970, soutenant que ceux-ci permettraient un processus décisionnel plus ciblé en réduisant le nombre de lieux de pouvoir autour du Cabinet. Heath a aussi créé le Central Policy Review Staff (CPRS), qui était chargé de transcender les frontières départementales (Flinders, 2002). Plus récemment, les gouvernements New Labour, notamment sous Blair, ont préconisé le « gouvernement décloisonné » ("joined-up government ») comme solution aux problèmes épineux de coordination (Lewis, 2009). Le New Labour a mis en place des structures innovantes (la Social Exclusion Unit, la Strategy Unit) pour tenter tout à la fois de traiter tous les problèmes liés à l'exclusion sociale, de créer de nouveaux instruments budgétaires et des unités spécialisées (Rough Sleepers Unit, Children \& Young People's Unit) capables d'imposer des priorités aux ministères réticents, et au total de renforcer la capacité de pilotage stratégique du Premier ministre. L'un des programmes interministériels qui a réussi, appelé «Sure Start », a consisté à regrouper les ressources de plusieurs ministères (Éducation, Emploi, Santé) se rapportant au « groupe-cible » des jeunes, un budget commun et 
un ensemble de règles institutionnelles favorisant la cohésion. Mais ces exemples de synergie interministérielle sont plutôt rares, si dépendants qu'ils sont d'un fort investissement de capital politique (une ressource qui diminue avec le temps) et du regroupement de ressources ministérielles jalousement gardées.

Notre enquête a mis en évidence des stratégies d'expérimentation organisationnelle dans chacun des pays observés. Mais les programmes de réforme de l'État ne sont pas construits sur une tabula rasa, et certainement pas dans les démocraties d'Europe occidentale complexes étudiées ici. Le beau récit du « gouvernement décloisonné » peut estomper les limites entre imputabilité (accountability) managériale et démocratique, peut menacer ou renforcer des intérêts fondamentaux. On peut aussi les interpréter comme des tentatives d'acteurs rivaux au cœur de l'État de contrôler la sphère de la coordination interministérielle, qui devient un enjeu toujours plus important car la formulation des politiques publiques est de plus en plus interministérielle et multi-niveaux dans les États membres de l'UE. Dans le cas britannique, les efforts visant à renforcer le pouvoir du Premier ministre vont à l'encontre de la fonction de coordination fondamentale du ministère des Finances, et une dynamique similaire s'observe en France (avec le ministère du Budget et des Comptes publics qui rivalise avec le cabinet du Premier ministre), en Espagne (où l'autorité du Premier ministre est régulièrement renforcée) et en Allemagne (où l'autorité du Chancelier est limitée par de puissantes traditions juridiques et départementales). En effet, le NMP semble être le dernier répertoire de légitimation utilisé par les concurrents impliqués dans ces compétitions de longue haleine pour élargir leur domaine et renforcer leur autorité au sein des exécutifs centraux.

Les effets de mode en matière de réforme sont ici une deuxième variable d'importance, qui remettent en question toute description trop statique de la longévité des formes institutionnelles. Les effets de mode, le benchmarking entre pays, les transferts internationaux de modèles d'action publique peuvent produire des récits de politiques publiques communs et un ensemble d'instruments d'action publique communs qui, à tout le moins, peuvent donner l'apparence de trajectoires de réforme convergentes. Les réformateurs publics, partout en Europe (et au sein de la Commission européenne), ont tendance à utiliser des discours de légitimation similaires s'inspirant du NMP et axés sur les «trois E », une «meilleure réglementation » ou la " démarche qualité ». Ces outils narratifs ont souvent été associés au recours à une boîte à outils commune, avec agencification, budgets de programme, contractualisation des conditions d'emploi et salaire à la performance (Hood, 1998). La récente littérature comparée sur les instruments d'action publique fournit des éléments empiriques sur la force des tendances organisationnelles convergentes dans divers domaines (Lascoumes et Le Galès, 2004). Dans les cas précis examinés ici, l'on observe que ces modes réformatrices semblent faire davantage sens dans certains contextes plutôt que dans d'autres, et qu'elles sont réinterprétées à la lumière des traditions de chaque État et des formes organisationnelles, quand elles ne sont pas simplement des discours commodes (Dyson, 2010). D'autre part, les tendances convergentes vers la mise en place de vastes « super-ministères » soi-disant omniscients et tout-puissants s'inscrivent aussi dans le cadre d'une évolution générale qui s'efforce d'apporter une réponse institutionnelle à des revendications politiques en matière de gouvernement décloisonné. 
En forme d'illustration, examinons brièvement le cas de l'agencification pour démontrer en quoi c'est une mode et en quoi son contenu substantiel varie considérablement en fonction des cultures politico-administratives nationales, des contextes et des configurations de forces. Même si des agences sont apparues partout ou presque, leurs formes, et notamment le degré d'autonomie de ces entités à l'égard des ministères centraux, sont extrêmement variables d'un pays à l'autre en Europe. Dans certains pays, cette délégation de responsabilités réglementaires et de prestation de services à des organismes publics autonomes remonte à plusieurs siècles, par exemple dans le remarquable modèle suédois de l'Ambetsverk, et n'a aucun lien avec les arguments économiques néolibéraux. Au Royaume-Uni, après la période des années 1960-70 durant laquelle Conservateurs et Travaillistes étaient favorables à des ministères de grande envergure et diversifiés, le Gouvernement Thatcher a introduit la mode propre au NMP consistant à réduire les effectifs de ministères centraux réaxés sur des missions stratégiques et à transférer massivement la prestation de services publics à des agences exécutives, mais sans prendre le risque de leur conférer une autonomie équivalente à celle dont jouissaient les agences suédoises (à propos du système suédois et de ses évolutions limitées, voir Pierre, 2003). Quelques années plus tard (comme observé plus haut) le Gouvernement Blair, sans reconsidérer la nouvelle structure « agencifiée » de l'administration publique, dénonçait la fragmentation excessive et les problèmes de coordination créés par la multiplication des agences : la nouvelle mode est devenue le « gouvernement décloisonné ». Dans d'autres cas, la situation est différente, par exemple en France, où des « établissements publics » plus ou moins autonomes existent depuis le XIXe siècle : la mode des agences a été adoptée en France bien plus récemment qu'au Royaume-Uni ou aux Pays-Bas, et dans le but de renforcer - et non d'affaiblir ! - le rôle de l'État dans des domaines politiquement sensibles, comme la sécurité sanitaire et alimentaire. La situation est aussi très complexe dans le cas de l'Allemagne, où la doctrine du ressortprinzip intègre une éthique de responsabilité politique pour les actes administratifs, ce qui est difficile à concilier avec une gestion autonome, mais où un véritable "zoo » d'entités publiques hétérogènes s'est constitué au fil du temps et ne s'est jamais simplifié.

Notre enquête a aussi mis en évidence des logiques hybrides d'ingénierie institutionnelle. L'une des observations empiriques les plus marquantes, dans ces études comme en d'autres similaires est que deux tendances différentes et d'apparence assez contradictoire ont coexisté dans les États membres de l'UE en ce qui concerne les réorganisations administratives : I'agencification d'une part, et les fusions administratives d'autre part. Au lieu d'attribuer des qualités sui generis à ces modèles institutionnels, ou de les considérer comme relevant de types d'État spécifiques, ou comme représentant pour l'essentiel des tendances transnationales globales, nos études de cas apportent des preuves empiriques de ce que ces modèles opèrent le plus souvent dans le cadre de nouvelles formes d'hybridation institutionnelle. Les agences et les fusions visent à répondre à des objectifs de spécialisation et de déspécialisation, qui peuvent coexister dans des cadres administratifs et des systèmes politiques très variables, y compris les cas examinés ici. En d'autres termes, un processus plus large d'hybridation institutionnelle est à l'œuvre, qui peut conjuguer les innovations institutionnelles résultant de transferts transnationaux et la persistance de structures et de pratiques endogènes, et qui implique les formes organisationnelles, comme les agences et les regroupements, 
en fonction des priorités du moment. Les fusions comme l'agencification sont justifiées de façon routinière par référence à un discours global sur la productivité de l'État ; les deux permettent aussi, de manières différentes, à des enjeux et secteurs d'action publique particuliers (comme la sécurité alimentaire, la santé ou l'environnement) de bénéficier d'une meilleure mise à l'agenda.

Conformément à cette thèse de l'hybridation, on observe la coexistence de grandes tendances de spécialisation et de déspécialisation dans un même pays à une même époque, ainsi qu'une évolution au fil du temps. Le cas de la France est particulièrement illustratif à cet égard. Une première « vague » de création d'agences publiques a eu lieu dans le domaine de la santé publique, des médicaments et de la sécurité alimentaire au milieu des années 1990. Après un ralentissement visible, le rythme de création de nouvelles agences s'est accéléré entre 2002 et 2007 et, surtout, la nature des agences créées a évolué (Cole, 2008). En parallèle, une autre tendance, apparue depuis 2007, consiste à ramener certaines agences (mais pas toutes) sous le contrôle de l'administration de l'État. En effet, des organes formellement indépendants, quasi-publics, comme des entreprises publiques et les chambres de commerce, ont été intégrés dans le processus de planification centrale dans le cadre de la révision générale des politiques publiques, ce qui illustre l'influence du ministère du Budget et des Comptes publics. À côté de la spécialisation des agences, l'ère Sarkozy a innové avec la création de super-ministères, comme le MEEDDAT (le ministère de l'Écologie, de l'Énergie, du Développement durable et de l'Aménagement du territoire). Même si le MEEDDAT regroupe quatre anciens ministères au sein d'un ministère unique, le nombre de directions générales est passé de 35 à cinq seulement. Ce qui unit ces évolutions apparemment contradictoires est le discours global sur la productivité de l'État et l'objectif stratégique du renforcement des capacités de l'État. La révision générale des politiques publiques (RGPP) du gouvernement Fillon a apporté un cadre général et une justification pour réduire les coûts, allant du non-remplacement de la moitié des agents de l'État partant à la retraite à la fermeture d'hôpitaux de campagne, de bureaux de poste, de centres fiscaux et à la restructuration globale du système judiciaire français. La révision générale des politiques publiques a aussi apporté le cadre général nécessaire pour d'importants regroupements bureaucratiques, en particulier celui survenu au sein du ministère des Finances entre les administrations autrefois rivales (et les innombrables agents qu'elles employaient en province) responsables du calcul des impôts (la Direction générale des impôts, DGI) et du recouvrement de l'impôt (la Direction générale de la comptabilité publique, DGCP), une division qui remontait à l'ère napoléonienne. Si les considérations d'économie politique sont importantes dans ces regroupements, la volonté de renforcer les capacités de l'État qu'ils représentent l'est encore plus. Le fait de regrouper deux administrations présentant des intérêts syndicaux profondément ancrés, comme dans le cas évoqué plus haut, vise à renforcer un leadership politique redoutant que des intérêts organisés ne mettent la main sur la bureaucratie. Ces exemples de fusions stratégiques pourraient se multiplier durant la présidence de M. Sarkozy, par exemple avec la création d'un nouveau service de l'emploi unifié (Pôle emploi) à partir de deux services autrefois autonomes (I'ANPE et les ASSEDIC). Ces exemples ont tous un point commun : une direction stratégique de l'exécutif central et une volonté d'exercer une forme de leadership politique sur le processus de réforme de l'État. 
Quatrièmement, la dynamique interactive entre les stratégies collectives des institutions et groupes concernés est évidente dans chacun de nos exemples nationaux. Des études de cas dans le domaine apparemment technique des réorganisations administratives font ainsi apparaître des logiques institutionnelles et des stratégies très variées dans chaque pays, dans chaque secteur. Le fait de regrouper des départements ministériels, des directions générales et d'autres organes administratifs (comme des corps), ou de scinder l'exécutif central ne découle jamais d'une logique neutre fondée sur l'optimum de Pareto. II est évident que les réformes administratives visent non seulement à améliorer les résultats, mais aussi à défendre certains intérêts essentiels aux dépens d'autres ou à mieux faire connaître des questions/domaines sensibles. Par exemple, les réformes qui fragmentent le secteur public en diverses agences spécialisées (comme les Next Steps au Royaume-Uni) visent parfois à expérimenter de nouvelles formes de direction centrale, à affaiblir l'élite des « mandarins de Whitehall » en offrant d'autres sources d'expertise à la bureaucratie traditionnelle ou à renforcer l'importance politique de questions stratégiques spécifiques. Les réformes axées sur la décentralisation territoriale ou sur la délégation de pouvoirs (devolution), qui renforcent l'autonomie des gouvernements régionaux et locaux, peuvent avoir une logique instrumentale similaire. D’autre part, les élites administratives de l'exécutif central proposent parfois et gèrent parfois elles-mêmes les regroupements administratifs ou les réorganisations qui visent à renforcer leur influence stratégique - des observations qui cadrent avec les affirmations fondamentales des célèbre modèles de « maximisation budgétaire » ou de « bureau-shaping » (Niskanen, 1971; Dunleavy, 1991). En somme, nos cas sont une validation empirique du fait que les regroupements et autres formes de remaniements administratifs interviennent, comme toutes les politiques publiques, dans le cadre de jeux de pouvoir relevant des « bureaucratic politics » entre des rationalités institutionnelles multiples, incohérentes et contradictoires (comme l'indiquent Allison et Halperrin dans leur classique, 1972), qui débouchent sur la victoire de certaines institutions et de certains groupes concernés, alors que les autres sont perdants ou victimes.

Enfin, une dynamique multi-niveaux intervient dans trois de nos cas au moins. Le cas espagnol associe la croyance persistante dans un État unitaire au centre et une détermination tout aussi forte des Communautés autonomes qui souhaitent développer leurs propres formes de capacité politico-institutionnelle, une entreprise couronnée de succès en Catalogne (Heywood et Molina, 2000; Parrado, 2008). À l'instar de l'Espagne, en quelque sorte, le Royaume-Uni associe des principes unitaires d'organisation territoriale, tout en conservant un État central limité mais puissant et une fonction publique théoriquement unifiée. Dans le cas britannique cependant, l'exécutif central a à peine été affecté par le développement de puissants gouvernements décentralisés - c'est du moins ce qu'il semblerait. Dans le cas de l'Allemagne, le modèle de fédéralisme coopératif produit un degré élevé d'échanges multiniveaux, d'influences croisées, d'échanges de connaissances et de négociations. C'est probablement en France que la dynamique multi-niveaux s'est avérée la plus faible, puisque c'est un pays où les processus de réforme de l'État central et de décentralisation ont toujours été séparés. Mais la situation évolue à présent : conformément à son approche en matière de regroupements administratifs, on observe que le Président Sarkozy a ouvertement invoqué, dans le cadre de l'instrument qu'est la révision générale des politiques publiques, des arguments axés sur la productivité 
pour intervenir directement dans la réorganisation non seulement de l'État territorial français (le regroupement des services « déconcentrés » de l'État dans les régions et départements), mais plus généralement pour réorganiser les gouvernements locaux et régionaux, notamment par le biais de changements importants dans leur système électoral (mis en œuvre depuis 2010), d'une réforme en cours de leurs finances (la taxe professionnelle) et de l'ouverture (par la Commission Balladur, 2009) d'un débat sur la « rationalisation » nécessaire du « millefeuille » institutionnel des gouvernements régionaux et locaux, qui pourrait au final déboucher sur la suppression des départements et le regroupement de leurs compétences et de leurs bureaucraties avec celles des régions d'une part, et des communautés urbaines de l'autre.

\section{Pour conclure...}

Comme cela a souvent été le cas avec toutes sortes de solutions à la mode adoptées sous la pression politique au cours de diverses périodes de l'Histoire en ce qui concerne la structure de l'État (au sens large), il semble que les résultats concrets et l'impact social, politique et économique concret-réel de ces regroupements administratifs et autres réorganisations tellement à la mode à l'heure actuelle dans les pays d'Europe et de l'OCDE, tels ceux présentés et examinés dans le présent symposium, soient encore relativement difficiles à évaluer avec précision (comme l'a récemment indiqué Pollitt, 2009), et s'avèrent peut-être plus modestes que prévu (comme l'illustre notamment un rapport du National Audit Office britannique, Reorganising central government, 2010, qui donne à réfléchir).

La prudence est également de mise lorsqu'on examine les regroupements administratifs et les réorganisations dans les États membres de l'UE du point de vue global de la diffusion du NMP en tant que forme transnationale de convergence idéationnelle et " praxéologique », même en tenant compte des variations nationales et sectorielles quant à son interprétation et sa mise en œuvre. Même s'il est indéniable que l'agencification et les regroupements administratifs sont plus ou moins justifiés par référence aux mêmes explications et répertoires d'action publique globaux, il ne faut pas oublier que ces fusions et réorganisations, qui sont une caractéristique traditionnelle de la gouverne des appareils administratifs, existaient déjà bien avant l'ère du NMP (comme le démontre Pollitt, 1984).

En outre, nos observations n'offrent guère d'éléments probants de purs et simples phénomènes de mimétisme institutionnel (Mény, 1993). Au contraire, et conformément aux versions plus sophistiquées de la littérature sur les transferts de politiques publiques (par exemple, Dolowitz, 2000), nos études de cas montrent clairement la mesure dans laquelle certaines caractéristiques communes sont ancrées de manière particulière dans chaque État, chaque niveau territorial de gouvernement, chaque secteur de politique publique, chaque contexte localisé. Même s'il existe des récits de politique publique et des répertoires d'instruments transnationaux communs, les articles présentés dans ce numéro suscitent de sérieux doutes quant à savoir si les pressions communes en faveur d'un transfert de politiques produisent des formes organisationnelles vraiment convergentes. II n'y a pas qu'en Asie que les réformateurs nationaux « sélectionnent les items dans un menu » (Turner, 2002). Une fine analyse empirique des réorganisations administratives, réalisée dans certains pays de l'UE, remet clairement en question les visions trop déterministes du NMP, 
comme celles annonçant un « démantèlement des États démocratiques » (Suleiman, 2003). En somme, s'il existe bien des tendances communes en ce qui concerne les réformes administratives, ces tendances font l'objet de formes surprenantes « d'acclimatations » très variées, d'hybridations imprévues et donnent des résultats concrets souvent paradoxaux (comme l'indique Eymeri-Douzans dans une autre étude, 2008). Le gouvernement des systèmes démocratiques et de leurs bureaucraties publiques ne peut qu'être un « art de l'État » (Hood, 1998) imparfait - un « art » de gouverner consistant précisément à s'adapter en permanence au contexte et aux configurations « locaux », ce qui est une question de survie pour ceux qui sont au pouvoir. Un telle adaptation aux différents contextes nationaux/régionaux/sectoriels différents implique une différenciation concrète entre les trajectoires nationales/régionales/sectorielles de réforme.

\section{Références bibliographiques}

Allison, Graham et Halperin, Morton (1972). "Bureaucratic Politics. A Paradigm and Some Policy Implications", in R. Tanter, R. Ulman (eds), Theory and Policy in International Relations. Princeton: Princeton University Press: 40-79.

Cole, Alistair (2008). Governing and Governance in France. Cambridge: Cambridge University Press.

De Jong M. et al. (2002). The Theory and Practice of Institutional Transplantation. Dordrecht: Kluwer Academic Publishers.

Dolowitz, David (2000). Policy Transfer and British Social Policy. Learning from the USA? Buckingham: Open University Press.

Dreyfus, Françoise et Eymeri(-Douzans), Jean-Michel (eds)(2006). Science politique de l'administration. Une apprche comparative. Paris: Economica.

Dunleavy, Patrick (1991). Democracy, Bureaucracy and Public Choice. London: Harvester Wheatsheaf.

Dyson, Kenneth (2010). The State Tradition in Western Europe. London: ECPR Press.-Douzans, JeanMichel (2001). La fabrique des énarques. Paris: Economica.

Eymeri-Douzans, Jean-Michel (2008), "Les réformes administratives en Europe: Logiques managérialistes globales, acclimatations locales", Pyramides n 15, November: 71-93.

Eymeri-Douzans, Jean-Michel (2010). "Conclusion" in J.-M. Eymeri-Douzans and J. Pierre (eds). Administrative Reforms and Democratic Governance. London: Routledge.

Flinders, Matthew (2002). "Governance in Whitehall", Public Administration 80 (1): 51-75.

Hall, Peter (1993). "Policy Paradigm, Social Learning and the State", Comparative Politics 25(3): 275-296.

Heywood, Paul et Molina, Ignacio (2000). "A Quasi-Presidential Premiership: Administering the Executive Summit in Spain" in G. Peters, R. Rhodes, and V. Wright (eds). Administering the Summit. Administration of the Core Executive in Developed Countries. London: Macmillan: 110-133.

Hood, Christopher (1991). "A Public Management for all Seasons?". Public Administration 69(3): 3-19.

Hood, Christopher (1995). "Contemporary Public Management: A New Global Paradigm", Public Policy and Administration: 104-117.

Hood, Christopher (1998). The Art of the State. Oxford: Oxford University Press;

Lascoumes, Pierre et Le Galès, Patrick (2004). Gouverner par les Instruments. Paris : Presses de Sciences Po.

Lewis, Christopher (2009). "Devolution and Public Policy in Scotland and Wales: a joined-up governance approach and the cases of special educational needs and adult community learning", PhD dissertation, : Swansea University. 


\section{Revue Internationale des Sciences Administratives 76(3)}

Linz, Juan et Stepan Alfred (1996). Problems of Democratic Transition and Consolidation. Baltimore: Johns Hopkins University Press.

March, James et Olsen, Johan (1989). Rediscovering Institutions: The organizational basis of politics. New York: the Free Press.

Mény, Yves (1993). Les politiques du mimétisme institutionnel: la greffe ou le rejet. Paris: L'Harmattan.

Niskanen, William (1971), Bureaucracy and Representative Government. Chicago (III.): AldineAtherton.

Parradó, Salvador (2010) Le rôle du gouvernement central espagnol dans un État à niveaux multiples, Revue Internationale des Sciences Administratives, Vol 76 (3) : 495-514

Peters, B. Guy, Pierre Jon et King, Desmond S. (2005). "The Politics of Path Dependency: Political Conflict in Historical Institutionalism", The Journal of Politics, 67 (4): 1,275-1,300.

Pierre, Jon (2003). "Central Agencies in Sweden: A Report from Utopia", in C. Pollitt, C. Talbot (eds). Unbundled Government: A Critical Analysis of the Global Trend to Agencies, Quangos and Contractualisation. London: Routledge: 203-14.

Pierson, Paul (2004). Politics in Time. Princeton: Princeton University Press.

Pollitt, Christopher (1984). Manipulating the machine: changing the pattern of ministerial departments, 1960-1983. London: Allen and Unwin.

Pollitt, Christopher (2003) "Joined up Government: a Survey". Political Studies Review 1 (1): 34-49.

Pollitt, Christopher (2009). "Structural change and public service performance", Public Money and Management, 29(5): 285-291.

Pollitt, Christopher et Bouckaert, Geert. (2004). Public Management Reform. A Comparative Analysis. Oxford: Oxford University Press.

Rhodes R.A.W. et Dunleavy P. (eds 1995). Prime Minister, Cabinet and core executive. London: Macmillan.

Richardson, Jeremy et Jordan, Grant (eds) (1982). Policy Styles in Western Europe. London: Allen and Unwin.

Rose, Richard et Davies, Phillip (1994). Inheritance in public policy. Change without choice in Britain. New Heaven: Yale University Press.

Savoie Donald (1994). Thatcher, Reagan, Mulroney: In Search of a New Bureaucracy. Pittsburgh, PA: Pittsburgh University Press.

Schmidt, Vivien (2008). "Discursive Institutionalism: The Explanatory Power of Ideas and Discourse", Annual Review of Political Science 11: 303-326.

Suleiman, Ezra (2003). Dismantling Democratic States. Princeton, N.J.: Princeton University Press.

Turner, Mark (2002). "Choosing Items from the Menu: New Public Management in Southeast Asia", International Journal of Public Administration 25: 1493-1512.

UK National Audit Office (2010). Reorganising central government. London: National Audit Office. 